\title{
ANÁLISE COMPARATIVA dOS EFEITOS PROMOVIDOS PELA APLICAÇÃO DE TENS E TERAPIA MANUAL EM LOMBALGIA CRÔNICA BASEADO EM EVIDÊNCIAS CIENTÍFICAS
}

\author{
Paula Ferreira Caldas ${ }^{1}$ \\ Lauro Kiyoshi Kurauchi ${ }^{2}$ \\ Juscelene Moraes Benicchio ${ }^{3}$ \\ Gabriela Alejandra Moya Fernandez ${ }^{4}$
}

Resumo: Várias estratégias terapêuticas são empregadas no tratamento da dor lombar crônica, no entanto a efetividade e a comprovação científica das mesmas, não se mostram de comum acordo. Terapia Manual (TM) e a TENS são amplamente utilizadas no tratamento da dor lombar crônica, portanto, este trabalho tem como principio verificar a eficácia entre os métodos de TM e a TENS no tratamento da lombalgia crônica. Através de buscas de artigos científicos nos bancos de dados: Scielo, BIREME, LILACS, PubMed, periódico CAPES, plataforma PEDro, BVS, foram localizados 39 artigos dos quais 30 sobre o assunto TM e 9 sobre TENS na Lombalgia Crônica. A TENS apresentou resultados satisfatórios na melhora da dor em $100 \%$ dos artigos enquanto que os métodos de TM apresentaram 93,33\%. Ao término deste estudo, concluiu-se que novos trabalhos devam ser realizados dentro deste tema, porém, avaliando a eficácia de cada uma das técnicas de forma isolada.

Palavras-chave: Lombalgia; TENS; Terapia Manual; Dor Crônica.

\footnotetext{
${ }^{1}$ Faculdade de Fisioterapia/Kroton-Anhanguera Educacional, Brasil. E-mail: caldasp@hotmail.com.

${ }^{2}$ Faculdade de Fisioterapia/Kroton-Anhanguera Educacional, Brasil. E-mail: emaildolaurok@gmail.com.

${ }^{3}$ Faculdade de Fisioterapia/Kroton-Anhanguera Educacional, Brasil. E-mail: ju.benicchio@hotmail.com.

${ }^{4}$ Faculdade de Fisioterapia/Kroton-Anhanguera Educacional, Brasil. E-mail: gabi_amf@yahoo.com.br.
} 